\title{
All-Fiber Optical Parametric Oscillator, Pumped by an All-Fiber Yb-based MOPA
}

\author{
Gys J. Van der Westhuizen and Johan Nilsson \\ Optoelectronics Research Centre, University of Southampton, Highfield, Southampton, SO17 1BJ, UK \\ gvdw@orc.soton.ac.uk
}

\begin{abstract}
We report on an all-fiber PCF-based optical parametric oscillator, synchronously pumped by an all-fiber Yb-doped MOPA. A pump-to-anti-Stokes conversion efficiency of $8.6 \%$ is reached at a frequency-shift of $142 \mathrm{THz}$.
\end{abstract}

(C) 2010 Optical Society of America

OCIS codes: (190.4370) Nonlinear optics, fibers; (190.4970) Parametric oscillators and amplifiers

\section{Introduction}

Nonlinear wavelength conversion to short as well as long wavelengths remains an active field of research, due to the constant demand for laser-quality light at new wavelengths not directly accessible by a laser transition. Fiber optical parametric converters are of particular importance, since they allow for both up- and down-conversion with large frequency shifts [1], and since the fiber gain medium is potentially inexpensive and offers the possibility of a compact and robust all-fiber configuration. Specifically of interest is the case where a fiber optical parametric converter is pumped in the normal dispersion regime, since this allows for frequency-upconversion over several $\mathrm{THz}$ [2]. Recent work on optical parametric generators, operating in this regime, have reported pump-to-anti-Stokes conversion efficiencies of over 30\% [3,4], however using sophisticated pump sources due to the high threshold for conversion in this configuration.

In this respect, the alternative fiber optical parametric oscillator (OPO) looks like an attractive options [5], providing a lower threshold that facilitates pumping with much simpler pump sources and ultimately all-fiber configurations. To date, however, due to the chromatic dispersion of silica, experiments on all-fiber OPO cavities have been limited to the $1550 \mathrm{~nm}$ wavelength region [6]. Although photonic crystal fiber (PCF) enabled the demonstration of OPOs with virtually arbitrary pump wavelengths, an all-fiber cavity at wavelengths outside the $1550 \mathrm{~nm}$ region has not yet been reported.

In this paper, we report a ring-cavity PCF-based all-fiber OPO, pumped by an all-fiber master-oscillator power amplifier (MOPA) operating at $1079 \mathrm{~nm}$. The OPO emits at $715 \mathrm{~nm}$, i.e., well outside the wavelength range directly accessible by power-scalable fiber lasers. We reach what we believe to be the largest pump-to-anti-Stokes conversion efficiency, to date, for any fiber OPO operating at a frequency-shift in excess of $140 \mathrm{THz}$. The in-fiber conversion efficiency to $715 \mathrm{~nm}$ was $8.6 \%$, limited, we believe, by fiber inhomogeneities.

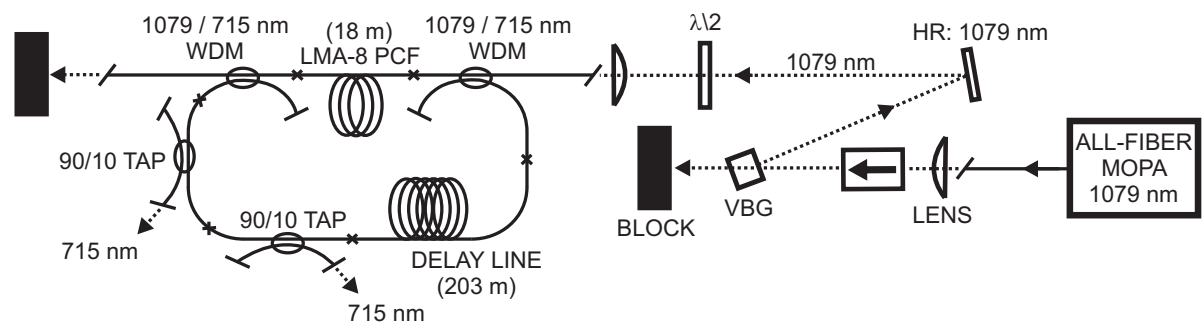

Fig. 1: Experimental set-up of the ring-cavity all-fiber OPO.

\section{Experiment and Results}

The all-fiber ring-cavity OPO is shown in Fig. 1. The linearly polarized output from the $1079 \mathrm{~nm}$ all-fiber pump source had a $1 \mathrm{~ns}$ pulselength and a pulse repetition frequency (PRF) of $1.73 \mathrm{MHz}$. Free-space coupling into the external cavity was necessitated by the requirement for a spectral filter (in this case, a volume Bragg-grating (VBG)). Filtering is crucial to avoid seeding of competing nonlinear processes such as Raman scattering. A large mode-area 
PCF (NKT Photonics LMA-8: $\Lambda=5.6 \mu \mathrm{m}$ and $d / \Lambda=4.9$ ) with a zero-dispersion wavelength of $1153 \mathrm{~nm}$ was chosen as the nonlinear converter. Both ends of the $18 \mathrm{~m}$ length of PCF were spliced to a WDM-coupler, to either combine or separate, the pump wave at $1079 \mathrm{~nm}$ and the anti-Stokes at $715 \mathrm{~nm}$. A delay line, consisting of $203 \mathrm{~m}$ of HI1060 fiber, ensured that the round-trip time of the OPO cavity could be matched to the pump's PRF for synchronous pumping, whilst two cascaded $10 \%$ tap-couplers were used to extract $715 \mathrm{~nm}$ light from the cavity. The cavity round-trip loss, dominated by PCF splice-loss and fiber loss in the delay line, was estimated to $6 \mathrm{~dB}$.

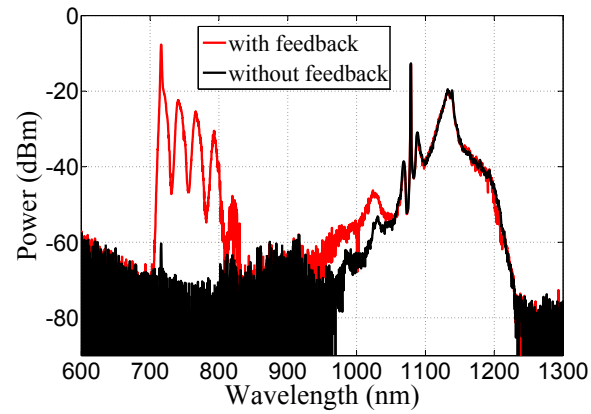

(a)

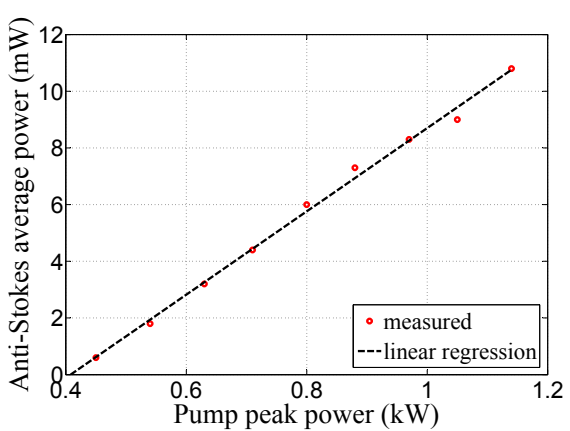

(b)

Fig. 2: (a) Influence of synchronized feedback on the OPO, as measured from the first tap-coupler (spectral resolution is $1 \mathrm{~nm}$ ). (b) OPO output power as a function of pump peak power, as measured from the second tap-coupler.

Fig. 2(a) shows the OPO output from the first tap-coupler, for the cases with and without synchronized feedback, at a peak pump power of $0.45 \mathrm{~kW}$ launched into the PCF. In the absence of synchronization, the OPO effectively functions as an OPG. The single-pass anti-Stokes power at $715 \mathrm{~nm}$ increases by over $53 \mathrm{~dB}$ upon synchronization of the feedback. The anti-Stokes peak is accompanied by several orders of stimulated Raman scattering (SRS) in the wavelength range $730-830 \mathrm{~nm}$. The generated SRS are pumped by the parametric anti-Stokes as the wave propagates through the delay fiber. Additionally, the $1079 \mathrm{~nm}$ pump generates a SRS Stokes $(1132 \mathrm{~nm})$ and anti-Stokes $(1024 \mathrm{~nm})$ as well as polarization modulation instability at a frequency-shift of $2.6 \mathrm{THz}$. These competing processes are clear indications that the parametric process, which would otherwise dominate, is suffering from inadequate phase-matching.

The use of cascaded tap-couplers resulted in strong attenuation at both $1079 \mathrm{~nm}$ and $1131 \mathrm{~nm}$. It was therefore possible to measure the average power, contained at the anti-Stokes and its associated Raman orders, directly from the second tap-coupler. These average powers are given as a function of pump peak power in Fig. 2(b). The anti-Stokes power reaches $10.8 \mathrm{~mW}$ at a maximum pump power of $1.14 \mathrm{~kW}$. The associated total anti-Stokes output power from both couplers is $22.8 \mathrm{~mW}$, which translates to $170 \mathrm{~mW}$ at the PCF output. We can therefore deduce that the PCF converts $8.6 \%$ of the pump input power to anti-Stokes power. To the best of our knowledge, this result represents the highest fiber OPO conversion efficiency, for such a large frequency-shift, to date.

\section{Conclusions}

We have demonstrated an all-fiber PCF-based OPO using a simple all-fiber pump source with peak powers readily achievable with conventional robust fiber technology. Synchronous pumping in the normal dispersion regime resulted in a pump-to-anti-Stokes conversion efficiency of $8.6 \%$ at a frequency-shift of $142 \mathrm{THz}$. We believe that the conversion efficiency is currently limited by inadequate phase-matching, likely caused by longitudinal inhomogeneity in the PCF, and that further power scalability is possible with an improved fiber.

\section{References}

[1] R.H. Stolen and J.E. Bjorkholm, "Parametric amplification and frequency conversion in optical fibers," IEEE J. of Quantum Electron. QE-18, 1062-1072 (1982).

[2] J.D. Harvey, R. Leonhardt, S. Coen, G.K.L. Wong, J.C. Knight, W.J. Wadsworth, and P.St.J. Russell, "Scalar modulation instability in the normal dispersion regime by use of a photonic crystal fiber," Opt. Lett. 28, 2225-2227 (2003).

[3] D. Nodop, C. Jauregui, D. Schimpf, J. Limpert, and A. Tünnermann, "Efficient high-power generation of visible and mid-infrared light by degenerate four-wave mixing in a large-mode-area photonic-crystal fiber," Opt. Lett. 34, 3499-3501 (2009).

[4] L. Lavoute, J.C. Knight, P. Dupriez, and W.J. Wadsworth, "High power red and near-IR generation using four wave mixing in all integrated fiber laser systems," Opt. Express 18, 16193-16205 (2010).

[5] W. Margulis, and U. Österberg, "Four-photon fiber laser," Opt. Lett. 12, 519-521 (1987).

[6] G.K.L. Wong, S.G. Murdoch, R. Leonhardt, J.D. Harvey, and V. Marie, "High-conversion-efficiency widely-tunable all-fiber optical parametric oscillator," Opt. Express 15, 2947-2952 (2007). 